Finally, the writer of the article appeals for protection for women in childbirth against the ill judged enthusiasm of the untrained obstetrician.

It is evident that Caesarean section, which Sacombe thought he had put an end to in 1798, has shown marked evidence of revival, and his hydra has developed "heads" which were non-existent in his day. The performance of Caesarean section for cases of placenta praevia, accidental haemorrhage, malpresentations, and eclampsia is being overdone, though not to the same extent as for "contracted pelvis."

It is sad that such a beneficial operation as Caesarean section, which has been developed to such a pitch of perfection that a woman, however deformed, can be delivered with safety to herself and child, should be brought into discredit by being performed in cases for which it is unnecessary.

\section{Antenatal Diagnosis.}

I have endeavoured to show some of the changes which have occurred in the treatment of contracted pelvis in the lifetime of the Medical Society of Iondon. Further improvement of the treatment depends on more accurate measurement of the pelvis and foetal head, probably by means of $x$ rays. Every woman should have her pelvis measured to see if she is fitted for child-bearing. During pregnancy she should be examined at the thirty-fourth week to ascertain the presentation of the child and its size relative to that of the pelvis. If the pelvis is contracted, it should be treated by one of the methods suitable for the particular case. Of these methods at the present time, in my opinion, induction of premature labour is employed too little and Caesarean section too much. As opinions are of less value than facts I give the results of three years' practice at University College Hospital, where the routine mentioned above has been adopted.

In the three years (1920, 1921, and 1922) 5,647 women were delivered. Of these, premature labour was induced tor contracted pelvis 113 times, all the mothers and 101 of the children surviving. Caesarean section for contracted pelvis was performed 32 times, all the mothers and 30 children surviving. Craniotomy was performed 4 times (twice for dead children and twice for hydrocephalus); the four mothers recovered. Forceps were used (for various reasons) 168 times (that is, in less than 3 per cent. of the cases), all the mothers recovering. I am indebted to the obstetric registrar, Mr. Leslie Williams, for these figures.

\section{Remaining Risks of Caesarean Section.}

Caesarean section entails risk of hernia, of adhesions, and rupture in subsequent pregnancy. These risks are probably avoidable in aseptic cases by proper suturing and sutures. How great these risks are can only be ascertained when obstetricians publish all their cases instead of a selection of cases done in favourable conditions before or in the early stages of labour, other cases being treated by craniotomy or hysterectomy. The sterilization of a woman after Caesarean section in an uncomplicated aseptic case is, in my opinion, not justifiable; in complicated cases hysterectomy is sometimes required.

\section{Concluston.}

In conclusion, I hope I may be pardoned for bringing a somewhat technical subject before the Medical Society of London. In the past some hard things have been said of obstetricians; in recent days, however, they have been commended as integrators of medicine. Agreeing with Sir Clifford Allbutt as to the importance of that integration, I have placed my views before a Society which by its constitution and practice is the best example of the advantage to be derived from discussion of medical subjects $1 . y$ physicians, surgeons, and general practitioners. With the assistance of the medical and surgical secretaries, of whose devotion to the interests of the Society I have already evidence, I hope to be kept mindful of that integration at the meetings over which it will be my privilege to preside. the British Empire. 1906.

\section{THE REGULATING AND REFLEX PROCESS.*}

\section{BY}

P. T. HERRING; M.D.,

PROFESSOR OF PHYSIOLOGY, ST. ANDREWS UNIVERSITY, AND PHYSIOLOGIST TO THE ST. ANDREWS INSTITUTE FOR Clinical Researcir.

\section{PART II.-THE NERVE IMPUISE: THE IMPORTANCE OF THE RECEPTORS: AND THE PRODUC- TION OF PAIN.}

THE anatomical unit of the nervous system, the neurone, consists of a nucleated cell body and one or more processes, one of which is always an axis cylinder. The other processes, when present, are dendrons, and frequently break up into numerous small branches known as dendrites. The function of the whole unit-dendrons, cell body, and axis cylinder - is to conduct the nerve impulse. The conducting element is a watery fluid containing protein and lipoids in the colloidal state, and possessing the physico-chemical attributes of living matter in this condition. No fibrils can be seen in the living neurone, though they appear in the dead nerve after certain methods of fixation and staining have been employed. The cell body and dendrons frequently contain a store of reserve energy in the form of nucleo-protein, which, when coagulated by certain fixatives and suitably stained, gives rise to the so-called Nissl bodies. The nucleus presides over the nutrition of the whole neurone and maintains it in a condition suitable for carrying the nerve impulses. But the presence of the nucleus and cell body is not essential for the passage of the nerve impulse, for, if cut off, the remainder of the neurone can still conduct for a limited time before it dies.

The nerve impulse, when excited by artificial means in an isolated nerve fibre, travels in both directions from the point of stimulation, but in the intact nervous system the nerve impulse travels normally in one direction only. The synapses between the neurones are irreciprocal, possessing a valve-like action which prevents any accidental impulse that may have been carried in the wrong direction from passing into the next neurone. The synapse only allows a nerve impulse to pass from the endings of the axis cylinder of one neurone to a dendron or cell body of the next neurone. Consequently the nerve impulse can only take the one direction through a physiological unit, and the neurone, when in a state of activity, only transmits its impulses in a forward direction.

The cell bodies of the peripheral afferent neurones are found in the ganglia on the posterior roots of the spinal cord, and in the corresponding ganglia on the cranial sensory nerves. The peripheral nerve fibre of each cell, though it possesses an axis cylinder, is morphologically the dendron of what was originally a bipolar cell. This dendron runs directly into an axon at the $T$-shaped junction near the body of the nerve cell in the ganglion, and there is evidence that the nerve impulse passes directly over this junction. Some of the spinal ganglion cells have small intracapsular dendrons, and consequently nerve impulses can reach the neurone through them, in which case the cell body must also be concerned in conduction. But little is known about the function of the intracapsular dendrons of the spinal ganglia. Their presence, however, indicates that a certain amount of analysis and redistribution of nerve impulses may take place in these ganglia.

In the olfactory nerve the cell body of the first afferent neurone lies in the olfactory membrane itself, and its dendron is a specialized portion of the cell which forms a tiny projection on the surface of the membrane. In the auditory and vestibular nerves the cell bodies are bipolar, and the peripheral process of each cell, though really a dendron, forms arborizations among the cells of the sense organs which are developed as special receptors. Little is known about the afferent neurones of the viscera, but there

* The expense of this research was in part met by a contribution from the Medical Research Council. 
is reason to believe that they are similar to those of the other afferent neurones except that their cell bodies may be smaller and their processes finely medullated.

The afferent neurone conducts nerve impulses into the central nervous system. Its axis cylinder, after reaching the brain or spinal cord, as a rule divides into ascending and descending branches, which in their turn give off other branches ending in synapses with the dendrites of other neurones. The path taken by the nerve impulse in the central nervous system is partly determined by the anatomical distribution of the axis cylinder carrying it, and partly by the character and resistance of the synapses it meets.

\section{The Nerve Impulse.}

The nature of the nerve impulse is unknown. It is not an electrical current, but is immediately preceded by a temporary change in the electrical potential of that portion of the axis cylinder which is at the moment conducting the nerve impulse. Such change in the electrical potential is common to living tissues in a state of activity, and by suitable means can be demonstrated as a "current of action." The whole neurone does not go into activity simultaneously, but responds to a stimulus in a characte:. istic manner. The response takes the form of a nerve impulse which travels from the point of excitation at a definite rate of about 120 metres per second. The nerve impulses, though capable of following one another at a frequency of from 50 to 60 per second, cannot fuse because after the passage of each impulse the nerve fibre is for a minute fraction of a second in a refractory state, during which it is incapable of conduction.

The nerve fibre requires a supply of oxygen to enable it to maintain its power of conduction, but it shows no measurable sign of oxidation, no rise of temperature, and no fatigue, even after long-continued activity. Further, the individual nerve impulse, as shown by the work of Keith Lucas and Adrian, is practically invariable in strength. It obeys the law of " all or nothing." The weakest stimulus that is effective produces a maximal response in the neurone. In other words, there is no grading of the individual nerve impulse. The number of impulses carried in unit time may vary, but cannot exceed a maximum imposed upon the neurone by the length of the refractory period. The only exception to the constancy of strength of the nerve impulse lies in the fact that immediately after the refractory period there is a supernormal phase in the neurone during which the response is a little stronger. The nerve impulse is always of the same nature by whatever nerve it is carried. This principle is the basis of Müller's law of " specific energies" or " specific irritability." It means that the end-result of the stimulus of nerve is determined, not by the character of the nerve fibre, but by the nature of its ending.

If we assume that the neurone has no power of automaticity, and that it only conducts in a forward direction, we are driven to seek the origin of all nerve impulses in the receptors on the afferent side of the nervous system. Whether the first assumption is true or not for all parts of the nervous system, it is so as regards the production of normal sensation and of reflex action, and it provides a good working hypothesis for clinical purposes. The nature and site of the receptors become matters of special importance.

\section{The Receptors.}

The receptors are widely different in structure and possibly in the manner in which they convert the adequate stimulus into a nerve impulse. In some instances the receptor appears to be a part of the dendron of the first afferent neurone itself. In the olfactory mucous membrane the dendron of the olfactory cell forms a fine projection which is stimulated by chemical substances in solution on the surface of the olfactory membrane. In the retina the outer ends of the rods and cones, modified dendrons, are stimulated by light, and possibly the actual stimulus is again a chemical one, depending upon the decomposition by light of a photo-chemical substance in them or in their neighbourhood.

In other sense organs the receptors are not always a part of the neurone itself, but special organs with which the neurone comes into close association. In the cochlea and vestibular apparatus the receptors are hair cells which are adapted to respond to a mechanical stimulus, and their response is converted into a nerve impulse in the neurones. Now nerve fibres are comparatively inexcitable whereas receptors respond to forms of energy which have no effect upon nerve, and which may be infinitesimally small in amount. The receptor is specially adapted to respond to some particular form of energy, and it acts as a transformer of this energy, and when necessary adds energy of its own in order to produce the excitation of the neurone. The most effective stimulus of nerve is an electrical one of a very definite character. The nature of the optimal stimulus of nerve-the "characteristic" as it was named by Wallerhas been more exactly determined by Keith Lucas. It is not so much a matter of difference of electrical potential as of the rate at which the alteration in potential takes place. Changes in electrical potential of this nature occur in living structures when set into activity, and it is possible that this factor is made use of in the excitation of nerve by some receptors. The receptor is set into activity by an appropriate stimulus for which it is adapted by structure, the stimulus being of a physical or chemical nature, and the change of potential thereby induced in the receptor may be the actual cause of excitation of the adjacent nerve fibres.

The receptors of the body are divided into two main categories: the exteroceptors, which receive stimuli from sources of energy outside the body, such as light, sound, and pressure; and the interoceptors, which are affected by events taking place inside the body. The proprioceptors are an important variety of the latter, and are the receptors which are excited by the tension of muscle, ligaments, and tendons, and the action of gravity. The proprioceptors give origin to the reflexes concerned with the maintenance of muscle tone and the posture of the body.

Receptors are not provided for all forms of energy-as, for example, Roentgen rays and ultra-violet light-and unless appropriate receptors are present such forms of energy do not excite nerve impulses nor give rise to sensation or reflex action. They may exert an influence upon the tissues of the body, but not of a nature adequate to excite nerve impulses until damage to the tissues is actually done.

The same form of energy may excite different receptors, especially when it differs in degree. Heat affects the receptors in the skin, and gives rise to the sensation of heat. If the wave-lengths are appropriate they excite the receptors in the retina and the sensation of light results. The same stimulus may excite the production of more than one sensation. Heat applied to the skin may cause a sensation of heat and a sensation of pain; pressure the sensations of pressure and of pain. The two sensations may be ascribable to the action of the same stimulus upon different receptors, and the paths of the nerve impulses are then separate from their origin to their termination in the higher levels of the central nervous system. There are réceptors which, when excited by an adequate stimulus, give rise to nerve impulses producing reflexes, but which are not normally associated with consciousness. Many of the interoceptors are of this nature, and initiate reflexes which are unaccompanied by sensation. On the other hand, the receptors which are concerned with the production of sensation may at the same time initiate reflexes. In the "conditioned" reflex the same receptor probably serves for both sensation and reflex. In some instances the same receptor gives rise to a nerve impulse which almost immediately spreads into two or more afferent channels and is carried to different parts of the brain. Light falling upon the retina excites its receptors, and the nerve impulses set up pass by different paths to produce the sensation of light and the reflex movements of the pupil. The retina is extional in that it contains several short neurones ani intervening synapses, which allow an immediate spreading of the nerve impulses, but an example of an immediate spread of the nerve impulse is also found in the "axon" reflex. Certain receptors in the skin give origin to nerve impulses which pass to the central nervous system and at the same time spread along branches of the axis cylinder 
of the same neurone to inhibit the contraction of local arterioles. The same neurone is here clearly capable of conveying nerve impulses which have different end-results, and the same receptor must be the source of origin of tha nerve impulse. The nature of the ending of the neurone mainly determines the result of the nerve impulse, but it does not follow that all nerve impulses in the same neurone are equally effective in the production of each end-result.

The synapse and the receptive substance at the terminations respectively of the neurone in the axon reflex may be so adjusted that each responds to nerve impulses of different frequency. In the artificial stimulation of vasomotor nerves the frequency of the stimulus is an important factor in determining the character of the end-result when applied to a nerve trunk containing both vaso-constrictor and vaso-dilator fibres. The point of importance to the present argument is that the same receptor and the same neurone can be concerned with the production of more than one kind of end-result. It appears to us probable that by the branching of the afferent neurone in the central nervous system, and its communication with other neurones similarly branching, the necessary pathways are present for the production of many end-results from the stimulus of a single receptor at the periphery. That the nerve impulse in an afferent nerve does not normally spread beyond certain well defined limits is explainable by the resistance it meets with in the synapses in the central nervous system; but, as will be shown later, the resistance of the synapses can be altered by abnormal conditions and very great spreading of afferent nerve impulses may occur.

The receptors concerned with some forms of sensation are well known, but others-and these unfortunately some of the most important from the clinical aspect-have not been definitely located. The nature and site of the receptors in the skin for the senses of touch, tactile discrimination, heat, cold, pressure, and pain, are uncertain. The histological structures in the skin recognized as receptors have had various functions assigned to them by different observers. They are being made the objects of a special investigation in the Clinical Institute, and a discussion of them is beyond the scope of this article. With the exception of the proprioceptors, so well elucidated by Sherrington, even less is known of the nature of the interoceptors. Some of them can give rise to sensation, notably of pain, but most, if not all, are concerned with the initiation of reflexes.

Many afferent neurones form free nerve endings or arborizations, especially in epithelium, which present no indications of possessing any special form of receptors. Indeed the free nerve endings are themselves regarded as the receptors. They appear to be the only nerve endings in the cornea, and as the sensation evoked by stimulation of the cornea is one of pain it is naturally inferred that the free nerve endings are the receptors for pain. But other effects are produced by stimulation of the cornea-namely, reflex closure of the eyelids and lacrymation. If the cornea be lightly stimulated by a fine wisp of moist cotton-wool no pain is felt but the reflex is produced, and the only sensation is that of the reflex itself. A stronger stimulus, and one that is actually injurious to the epithelial cells on the surface of the cornea, provokes a stronger reflex accompanied by pain. A minute foreign body, if of a harmful nature by reason of its hardness or chemical composition, occasions pain of an intense and widespread character. Similar free nerve endings are found in the skin, but the living cells amongst which they ramify are protected iy the dead and horny cells of the outer layers. A stronger stimulus must consequently be applied to the skin in order to produce pain, and the sensation thus elicited is a mixed one because the skin is capable of other sensations. Any modification of the epithelium alters the reaction. The application of a mild irritant soon abolishes from the area affected all sensations of heat and cold and tactile impressions, leaving an epithelium which, like the cornea, responds to all stimuli by reflex action and by the sense of pain. The pain is very like that of the cornea, being unmixed with other sensations, widely spread and incapable of exact localization.
The condition of the epithelium is, in our opinion, of very great importance in the maintenance of function of the receptors in the skin, and we regard the epithelial cells as the true receptors and not the free nerve endings. The free nerve endings lie among living cells in the epidermis. In the epidermis these cells are intimately connected one with another by intercellular bridges of protoplasm. Injury to any cell probably involves a widely spread response, and it may be that the reaction of the cells to the injury furnishes the effective stimulus which results in pain. Whether the actual nerve endings contain a receptive substance or not is unknown. It is quite possible that they do, and that the afferent end of each element in a reflex arc is alike endowed with a material which has the specially developed property of excitability as distinguished from that of conductivity. Such an element is present in the receptor organ of the sense of smell and in the receptive substance of the effector organ of voluntary muscle. In our opinion too little account has been taken in modern researches on sensation of the importance of the receptor and its re-establishment with the ends of regenerating nerve fibres.

\section{The Sense of Pain.}

Many tissues and organs of the body are insensitive to stimuli which in the skin produce pain. This is not because of the lack of afferent nerves, but because the receptors present are not adequate to such forms of stimulation. Most of these tissues are productive of pain when the stimulus is of such a nature as to excite the receptors, although the pain may not be felt in the organ affected but is referred to some other situation which is normally capable of sensation. The only known receptors in voluntary muscle are the muscle spindles, which are chiefly concerned with the initiation of reflex action but may give rise to muscle sense. Muscle is insensitive to ordinary forms of stimulation which cause pain in the skin. Pain of varying degrees of severity can occur in muscle, but it is always associated with some degrees of activity of the muscle, and is probably due to some condition which exaggerates the normal adequate stimulus of the muscle spindles, or renders these receptors more sensitive. The fact that pain in muscle is found to be associated clinically with contraction of its fibres makes it probable that the muscle spindles are the receptors for pain in voluntary muscle. The normal contraction of muscle is painless, but becomes painful when the circulation of blood through it is interfered with.

Pain can also arise from cardiac and involuntary muscle although these tissues are insensitive to crdinary forms of stimulation. Nothing is known about the nature of the receptors in these structures. They appear to be primarily concerned with the production of reflex action, and by analogy one would assume that they are stimulated by the activity of the structures in which they reside. This is found to be so, for pain in cardiac and involuntary muscle is associated clinically with activity of the organs in which it occurs, though some other factor appears to be necessary such as a deficiency in blood supply. It is often stated that tension is the exciting factor in the production of pain. But the term "tension" is apt to be misunderstood in its application to muscle. The function of muscle is to produce tension; the contraction of muscle does not necessarily imply its shortening, but it does imply tension in the muscle, and this tension is probably the adequate stimulus for the receptors in it. The artificial tension of muscle produced by the distension of a hollow viscus gives rise to pain, but it probably does so by stimulating the contraction of the muscle to overcome the distension. Great care is necessary in making clinical observations on the production of pain in the viscera that the results of reflex action-for example, slowing of the heart, vascular changes, faintness, nausea, and other symptomsare not mistaken for pain.

We are of the opinion that pain in muscle, whether voluntary, cardiac, or involuntary, is always associated with the contraction of the muscle, but that some other factor or factors are necessary and tbat a deficient blood supply is one of the most important. 
We regard pain as a sensation which under certain conditions is superadded to the reflex, and the nerve impulses giving rise to pain we regard as excited by the same receptors and carried into the central nervous system by the same afferent neurones as those which bring about the reflex. Pain may be readily added to the reflex, as in the case of the afferent neurones from the cornea, or more rarely added, as in those from the heart muscle. The harmful stimulus, as has been shown by Sherrington, takes precedence of all others in bringing about its distinctive reflex, even in the spinal animal in which there can be no perception of pain. These observations appear to us to point to the reflex as the more important end-result of the harmful stimulus. In certain cases of injury to the spinal cord where areas of hyperalgesia are found in the skin the slightest stimulation of the latter results in pain. We cannot but conclude that here we are dealing with a central change, and that the nerve impulses excited at the periphery, which in the normal individual would cause no pain, now do so because of their more ready access to the pain tracts in the spinal cord. The actual cause of this increased passage of nerve impulses into the pain tracts appears to us to be explainable by a decreased resistance in the synapses between the endings of the afferent neurones and the dendrons of the neurones which constitute the pain fibres of the spinal cord. We know that the nerve impulses giving rise to pain are carried by fibres which decussate to the opposite side of the spinal cord soon after the impulses have reached the cord, and that this decussation is not made by the incoming afferent neuranes. Consequently there must be a synapse intervening between the incoming afferent neurone and the neurone which carries the impulses upwards on the opposite side of the spinal cord. This synapse, guarding as it were the entrance to the pain paths, is of great significance, for it interposes a resistance which is probably very varied for different afferent neurones, and is in each case susceptible to those changes which are characteristic of the synapses in general. So far as we know the afferent fibre on arrival in the lower level of the central nervous system gives off a number of branches and makes connexions with other neurones. Pathways are thus provided for a great many reactions.

It seems to us that neither pain from tissues endowed with common sensibility, nor from the viscera, requires special nerve fibres from the periphery, and that the analysis of the afferent nerve impulses takes place in the lower level of the central nervous system. Pain from tissues endowed with common sensibility becomes educated, capable of localization, and modified by other sensations. Pain from the viscera is comparatively rare, uneducated, unmodified by other sensations, and the nerve impulses which cause it, on coming into the lower level of the central nervous system, excite the same neurones in the pain paths and give rise to pain which is referred to those areas cf higher sensibility with which it has by education been associated.

There is, therefore, no necessity to postulate the separate existence of special receptors and special nerve fibres for the sense of pain. It is not indeed reasonable to assume that structures like the ureter and other viscera are provided with special receptors and special nerve fibres to meet a pathological event which may not occur for generations. The stimulus which is adequate to excite pain in the viscera or elsewhere may do far more than this. It sets up nerve impulses which spread widely in the lower level of the central nervous system, and may give rise to nausea, vomiting, sweating, vasomotor, cardiac, respiratory, and other reflexes, or even shock. Pain may or may not occur in association with any or all of these symptoms. Pain is a concomitant very often, but is not the cause.

Pain is one of the most important manifestations of ill health, and when it occurs is of special diagnostic value because the site of its excitation can be more or less definitely located. In this way a clue is given to the site and cause of the disturbance which is not to be obtained from the other symptoms occasioned by the spreading of the nerve impulses in the central nervous system.

\section{PELLAGRA IN CHILDREN IN ENGLAND.} (With Special Plate.)

BY

\author{
ROBERT HUTCHISON, AND DONALD PATERSON, \\ M.D., F.R.C.P., \\ PHYSICIAN, \\ M.B., M.R.C.P., \\ HOSPITAL FOR SICK CHILDREN, GREAT ORMOND STREET.
}

Ovr object in reporting two cases of pellagra in children is to draw the attention of clinicians in this country to the fact that pellagra is endemic in England.

The diagnosis, except in typical cases seen during the spring and summer months, when the rash is well marked, is not easy, and a considerable number of cases probably remain undiagnosed on this account. In 1913 and 1914 Dr. C. R. Box. described three cases of pellagra in children, two of them fatal. In one of these cases the pathology was described in detail by Sir Frederick Mott. All of these children had lived throughout life in England.

CaSE I.

Edna S., aged 6 years and 10 months, admitted to Great Ormond Street Hospital, April, 1923. The complaint was loss of walking power and shaking of the limbs. This had been noticed for six months. The family history appeared quite normal, no other person being affected. She was breast-fed for six weeks, then put on Nestlé's milk up to 11 months, since when she has had the same food as her parents. She had meat first at 3 years. She had always had plenty of milk, eggs, vegetables, and fruit. She was always fond of "cornflour" and would take nearly half a pint had lived at Little a healthy child up to the age of $3 \frac{1}{2}$ years. She had lived at Little Blackenham-ive miles beyond Ipswich-all her life except between the ages of 4 and $5 \frac{1}{2}$ years, when she had lived at Walthamstow. She had had "eczema " every year, starting in the spring. It was first noticed to occur after an illness, said to be "meningitis," at $3 \frac{1}{2}$ years of age. This illness lasted three months and was accompanied by fever and drowsiness; recovery was gradual. The rash appeared as the child got better. It appeared on
the hands, feet, neck, and face. It appeared on the knees last year for the first time. It usually lasted four to five months, appearing first in March. In July, 1922, the child complained of "giddiness," and began to fall about. This came on suddenly, the child falling down when out walking. She did not lose consciousness and the attack lasted one and $a$ half hours. A month later she had two similar attacks.

Present Illness.-The patient was comparatively well until six months ago, when she gradually ceased to walk more than a few steps, and tremor of her hands and arms developed. She walked with the aid of the furniture. She complains of pains in her head which seemed to start at the back of her neck and shoot over her head. Her eyesight failed and she could not recognize objects across the street. She had some frequency of micturition by day and night.

On admission, the child was miserable but well nourished. There were patches of dermatitis on the face, neck, hands and inner stirfaces of the arms, knees, and backs of the thighs. The tonsils were large and injected. There was incontinence of faeces. The were large and injected. There was incontinence of faeces. The and reacted to light. No cranial nerve palsy was noted. All her and reacted to light. No cranial nerve palsy was noted. All her deep reflexes were increased. The plantar reflexes gave a flexor
response, and the abdominal reflexes were not obtained. No ankle response, and the abdominal reflexes were not obtained. No ankle
clonus was elicited. There appeared to be a certain amount of anaesthesia to pin-pricks over the legs and abdomen, but there was no definite distribution and it was difficult to be sure. Her Wassermann reaction was negative. The cerebro-spinal fluid was normal. Her mental condition was very backward and she seemed to have the feeling that she was continually falling. Nothing definitely pathological was made out from an examination of the fundi. The urine contained a few cells and a trace of albumin. The urea concentration test showed a normal output of urea by the kidneys. The blood urea was normal : $37 \mathrm{mg}$. per $100 \mathrm{c.cm}$. The rash which was present had the appearance of a dermatitis produced by exposure to the sun. A test meal showed a well marked reduction exposure to the sun. A test meal showed a well marked reduction
of the total acidity of the gastric juice. No free hydrochloric of the total acidity
acid was present.

The patient was seen by Dr. L. W. Sambon, who stated that the distribution and character of the rash and nervous symptoms were typically those of pellagra. The child became much worse during died. Unfortunately no necropsy could be obtained.

\section{Case Ir.}

Helen S., aged 10 years and 9 months. Admitted to Great Ormond Street Hospital, July, 1922. The complaint was progressive mental deterioration and inability to walk, and in addition a rash on the hands, feet, face, and neck which came in the spring a rash on the hands, feet, face, and neck which came in the spring
and summer and disappeared in the winter. She has lived all her and summer and disappeared in the winter. She has lived all her life at Acton, a suburb of London, without leaving the district.
The mother and father are both alive and well. The patient is 